\title{
PEDIATRIC AND CONGENITAL HEART DISEASE
}

\author{
Original Studies
}

\section{Remodeling and Thrombosis Following Closure of Coronary Artery Fistula With Review of Management: Large Distal Coronary Artery Fistula-To Close or Not to Close?}

\begin{abstract}
Srinath T. Gowda, ${ }^{1 \star} \mathrm{MD}$, Thomas J. Forbes, ${ }^{1} \mathrm{MD}$, Harinder Singh, ${ }^{1} \mathrm{MD}$, Julie A. Kovach, ${ }^{2}$ MD, Lourdes Prieto, ${ }^{3}$ MD, Larry A. Latson, ${ }^{4}$ MD, and Daniel Turner, ${ }^{1}$ MD

Background: To evaluate postdiscovery outcome of coronary artery fistulae (CAF). CAF treatment sequelae and risk factors for coronary thrombosis have not been adequately evaluated. Methods: Outcome on follow-up of 16 patients with CAF was reviewed. Risk factors for adverse coronary events were assessed based on type, size, and treatment of CAF. Results: Median age was 10 years (0.01-56). Seven patients had large, four medium, and five small sizes CAF. Eight had proximal and 8 distal type CAF. There were 7 in the intervention group (IG) and 9 in nonintervention (NIG). In the IG, 1 had myocardial infarction (MI) $<24 \mathrm{hr}$ with distal thrombosis following large distal type CAF closure. Follow-up angiograms in 6 pts showed; decrease in conduit coronary artery size towards normal in 4,1 had discrete intimal stenosis, persistent coronary dilatation in 1 , thrombosis of residual proximal fistula segment without $\mathrm{MI}$ in 2 , evidence of revascularization in 2 and neovascularization in 1 patient. In the NIG, 6 of the 9 pts available for follow-up were asymptomatic. Angiogram available in 1 patient showed persistent coronary dilatation with partial closure. Conclusion: Post-CAF treatment sequelae include thrombosis and MI, revascularization, persistent coronary dilatation, remodeling, and decrease in conduit coronary artery size towards normal. The large size distal type of CAF may be at highest risk for coronary thrombosis post closure. The optimal treatment approach to various morphologies of CAF at various ages remains to be determined. $\odot 2013$ Wiley Periodicals, Inc.
\end{abstract}

Key words: coronary artery fistula; remodeling; thrombosis; treatment

\section{INTRODUCTION}

Coronary artery fistulae (CAF) are rare congenital coronary anomalies. Small CAF can be clinically silent and may close spontaneously [1-3]. Moderate to large CAF can be hemodynamically significant and are associated with complications [1,4,5]. Many patients are asymptomatic early after CAF closure. More detailed follow-up studies, however, have identified post CAF closure sequelae including persistent coronary dilatation [6], residual leak [6], thrombosis with or without myocardial infarction [6-12], coronary stenosis with perfusion defects [13], and rarely death [4,14]. The risk factors for early or late coronary events have not been adequately evaluated. Recent reports have sug-

\begin{abstract}
${ }^{1}$ Pediatric cardiology, The Children's Hospital of Michigan, Wayne State University, Detroit Medical Center, Detroit, Michigan

${ }^{2}$ Adult cardiology, Michigan Adult Congenital Heart Center, Wayne State University, Detroit Medical Center, Detroit, Michigan ${ }^{3}$ The Center for Pediatric and Congenital Heart Diseases, Cleveland Clinic Foundation, Cleveland, Ohio

${ }^{4}$ Pediatric cardiology, Joe DiMaggio Children's Hospital, Hollywood, Florida
\end{abstract}

Conflict of interest: Nothing to report.

*Correspondence to: Srinath T. Gowda, The Children's Hospital of Michigan, 3901 Beaubien Blvd, Detroit MI 48201-2119, USA. E-mail: sgowda@dmc.org

Received 11 September 2012; Revision accepted 7 October 2012

DOI 10.1002/ccd.24699

Published online 14 February 2013 in Wiley Online Library (wileyonlinelibrary.com) 
A.

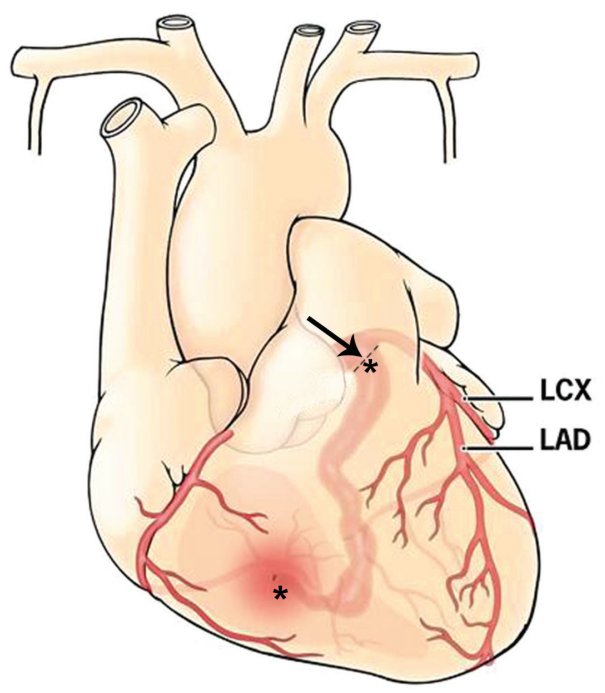

Fig. 1. Types of coronary artery fistula. A: Proximal type CAF: Proximal fistula arising from the central left main coronary artery. The arrow shows origin of the fistula and short segment proximal dilated left main coronary artery. B: Distal type CAF: Distal fistula arising from the distal major epicardial coronary artery (arrow). The

gested that large size, distal type CAF [9], and CAF draining to the coronary sinus [14] may be at higher risk for coronary thrombosis post closure. We sought to evaluate the clinical outcomes of various treatment options for CAF patients in intermediate to long-term follow-up.

\section{METHODS}

All patients who underwent evaluation for CAF treatment in the catheterization laboratory at The Children's Hospital of Michigan between January 1995 and Jan 2010 were identified after IRB approval. A retrospective review of all available medical records before and after CAF closure detailing history, anatomic, and functional evaluation of the coronary arteries, and use of anticoagulation was performed. To identify possible risk factors for thrombotic complications following closure, CAF were classified as proximal or distal [9]. In brief, the proximal CAF arises near the origin of a major epicardial coronary artery (Fig. 1). The short proximal segment of the feeding main coronary artery is dilated and the coronary artery distal to the fistula has normal caliber and branches. There are no significant coronary branches supplying the myocardium from the fistula itself. Distal fistula (Fig. 1) has their origin near the distal end of a major coronary artery. The conduit coronary artery proximal to a distal fistula gives rise to normal coronary branches that supply the myocardium. The increased
B.

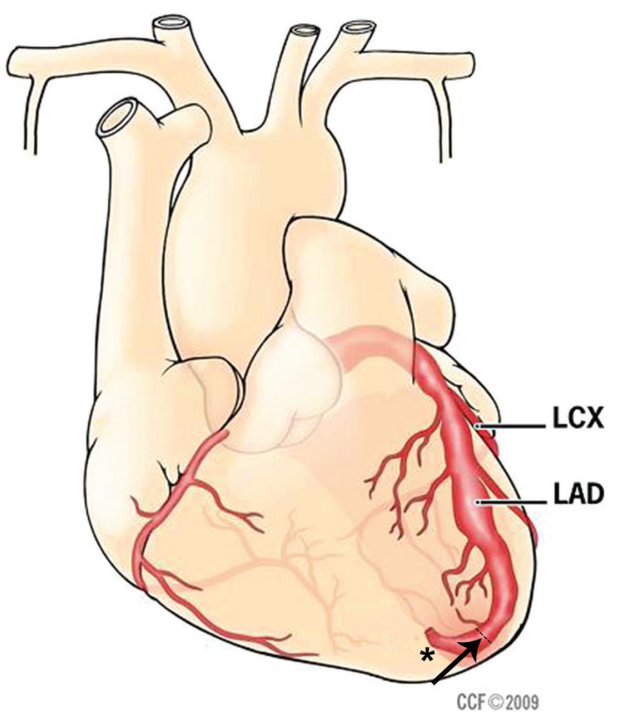

proximal conduit coronary artery is tortuous and dilated (LAD) with normal coronary branches supplying the myocardium. *Indicates sites of potential transcatheter or surgical closure. CAF, coronary artery fistula; LCX, left circumflex artery; LAD, left anterior descending artery. (modified copy, permission granted by Elsivier AJC).

flow in the feeding or conduit coronary artery (fistula flow plus normal myocardial flow) results in dilation and tortuosity of the conduit coronary artery proximal to the fistula. The sizes of the fistulae were categorized as small, medium, or large as per the descriptions in the catheterization reports. All CAF patients evaluated for treatment were categorized into intervention group (IG); who underwent either transcatheter closure (TCC) or surgical closure (SC) and Non-Intervention group; observation with or without anticoagulation.

\section{RESULTS}

A total of 16 patients were evaluated for CAF between 1995 and 2010. The demographics, CAF characteristics, treatment, and follow-up findings are detailed in Table I. The median age at closure was 10 years (0.01-56). All patients were asymptomatic except one infant presented with congestive heart failure due to a large CAF. Most CAF (13-16) drained into various right sided structures of the heart. The fistula origin was proximal in 8 and distal in 8 patients. The fistulas were graded as large in 7 , medium in 4 , and small in 5 patients. In the IG, TCC was performed in 6 and SC in one patient. All patients had complete occlusion without complications at the end of the procedure. Transcatheter closure techniques have been previously described [1,4]. Briefly, medium-sized fistula was closed with coils directly via an arterial approach. Large fistula was closed using occluder devices, 


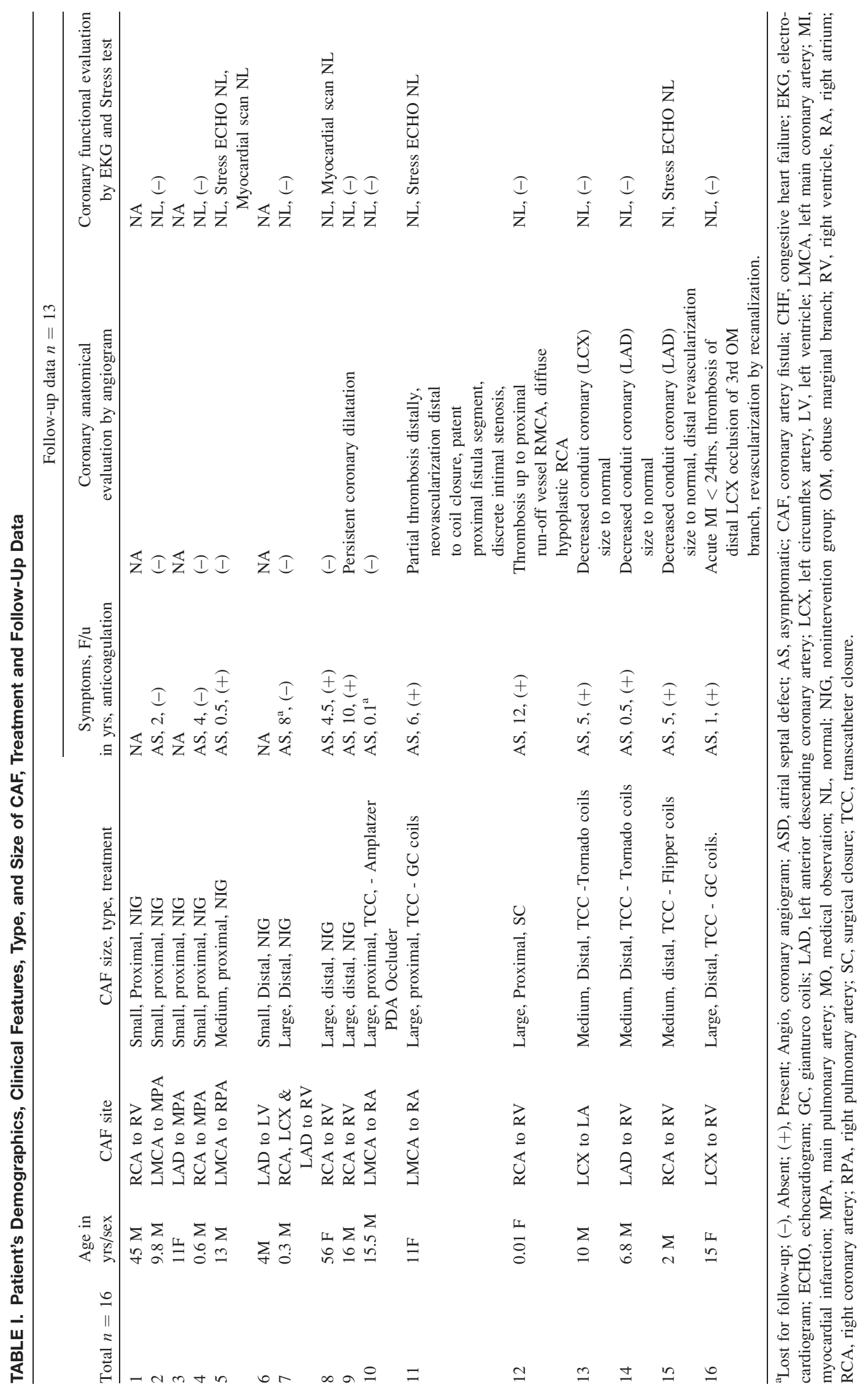



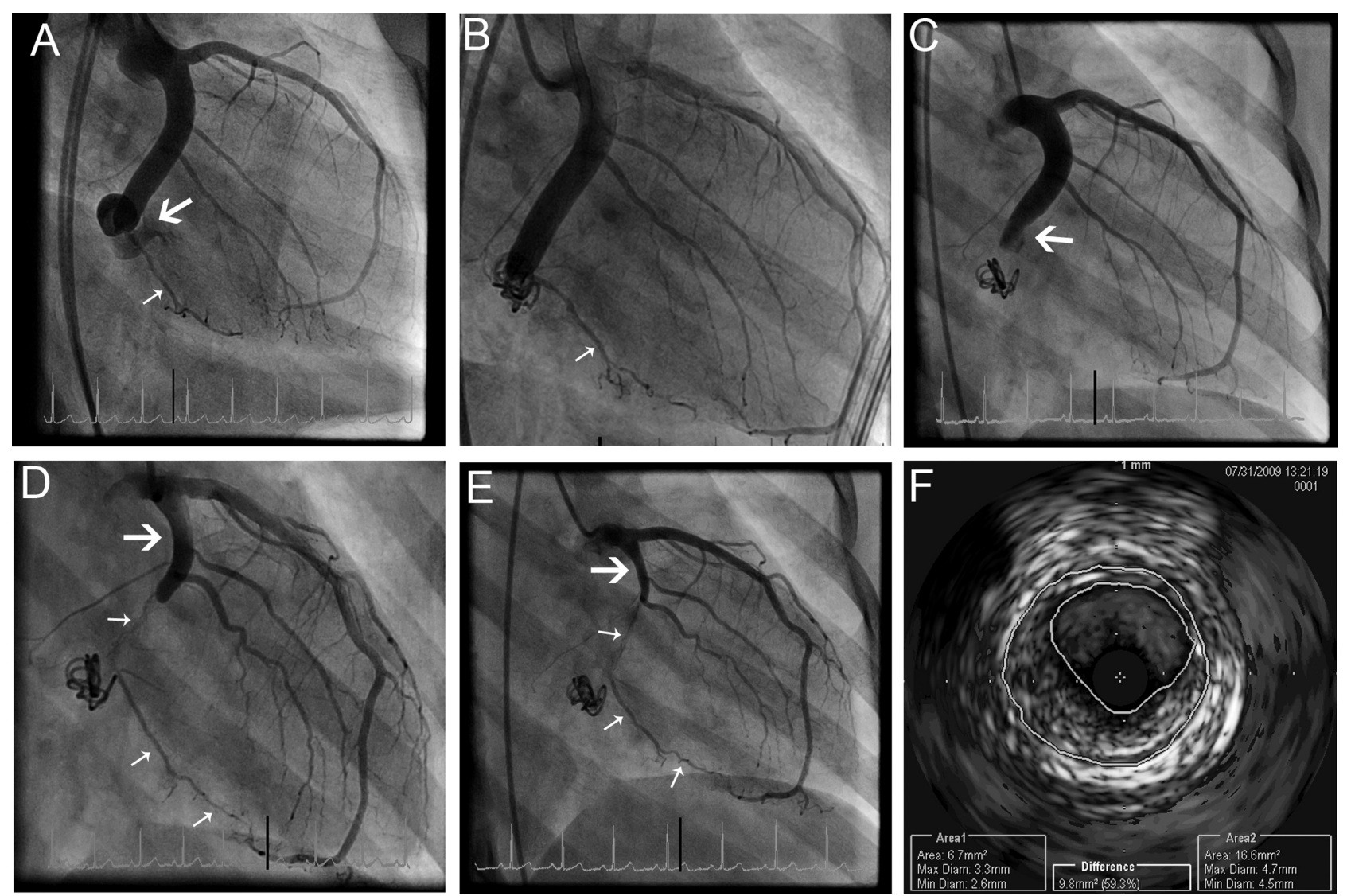

Fig. 2. Large distal fistula with thrombosis post closure. A: Large LCX to RV distal CAF in a 15-year-old female. Normal 3rd OM branch (small arrow) just proximal to the drainage point (arrow). B: Following coil closure, there is patent conduit LCX coronary artery with normal flow into 3rd OM branch. C: $<24 \mathrm{hr}$ post closure there was distal thrombosis with occlusion of the 3rd OM branch (arrow) causing acute MI. D. At 2-month follow-up, there was reduction in size of

preferentially from the venous route. For distal fistula extreme care was taken to place the occluding device as close to the actual fistulous opening as possible to avoid occlusion of proximal coronary branches supplying the myocardium. One patient had surgical ligation distally at or near the draining site under cardiopulmonary bypass. Nine patients comprised of a nonintervention group (NIG). Of these, 5 had a small CAF with no indication for intervention and in the remaining $4 ; 1$ with medium size proximal CAF and 3 large distal $\mathrm{CAF}$ had no intervention per physician and/or patient preference and was under observation with follow-up.

\section{CAF Sequelae Following Treatment on Follow Up}

A total of 13 patients were available for long-term follow-up with median of $3(0.1-12)$ years (Table I) and have remained asymptomatic. All patients $(n=7)$ in IG were available for follow-up, 4 had distal CAF.

proximal conduit LCX artery (arrow), revascularization of OM branch due to recanalization ( small arrows) E: Oneyear follow-up; remodeling with further significant reduction of conduit LCX artery (arrow) to normal. F. IVUS study showed uniform thickening of conduit LCX artery. CAF, coronary artery fistula; IVUS, intravascular ultrasound; LCX, left circumflex artery; RV, right ventricle; OM, obtuse marginal branch.

One patient (pt \# 16) had an acute coronary event within $24 \mathrm{hr}$ of TCC. She was 15 -year-old, asymptomatic with no history of hypertension, diabetes, hyperlipidemia, smoking, coagulation disorders or use of oral contraceptive pills with a large distal left circumflex (LCX) to right ventricle (RV) CAF (Fig. 2). Fistula closure with $8 \mathrm{~mm}$ Gianturco coil just distal to third obtuse marginal branch was performed and she was placed on oral ASA. At $12 \mathrm{hr}$ post occlusion she complained of severe chest pain, radiating to her shoulders associated with sweating. She had elevated serial Troponin levels. Her EKG and echocardiogram were normal without wall motion abnormality and had normal ejection fraction. She was treated with morphine, oxygen, glycoprotein IIb/IIIa inhibitors, heparin, and beta blocker. Cardiac catheterization within $24 \mathrm{hr}$ showed distal LCX thrombosis with complete occlusion of the third distal obtuse marginal (OM) branch and proximal extension of thrombus up to the second OM branch 


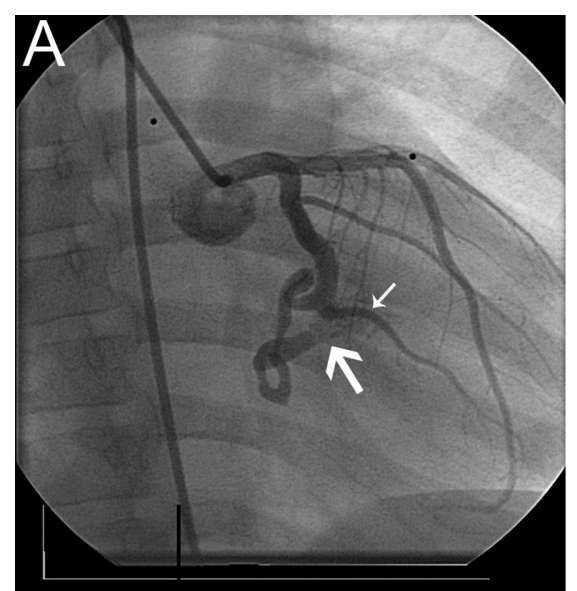

Fig. 3. Medium size distal CAF, LCX to LA. A: A 10-yearold with medium-sized distal CAF from the LCX to the LA (large arrow). The LCX artery distal to 2nd obtuse marginal (OM) branch - (small arrow) is tortuous. B: Post coil angiogram shows contrast in the distal LCX artery (small arrow), and there are small coronary branch (faintly profiled) from distal part of the LCX artery (small arrows). C: Follow-up 5
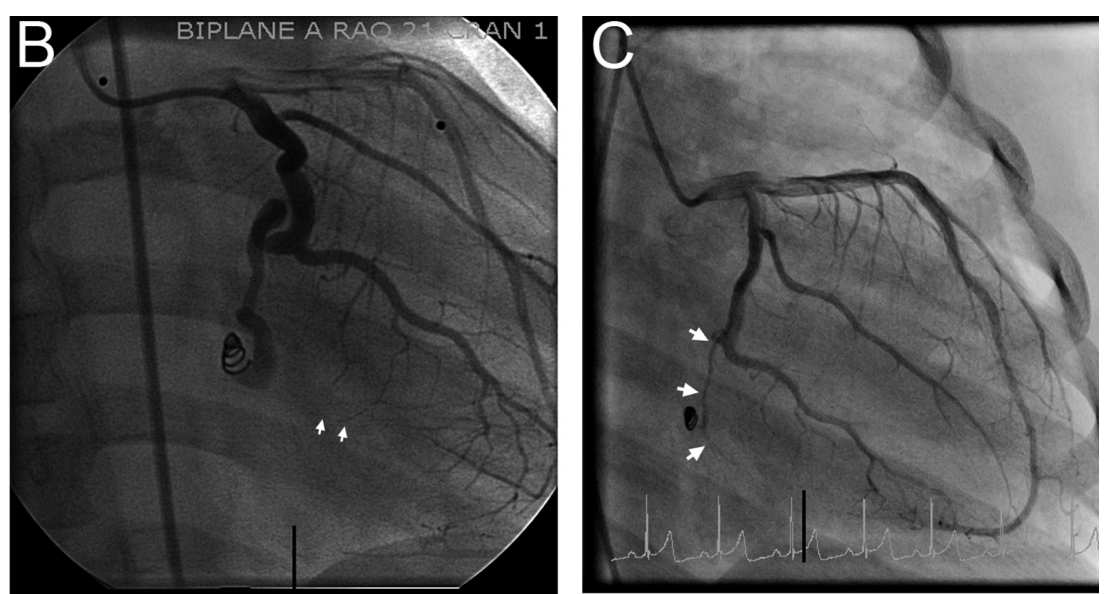

years later shows proximal conduit LCX artery (large arrow) has uniformly decreased in size (proximal to 2nd OM branch), significant reduction in distal LCX conduit artery with preserved flow to the small coronary branch (small arrows). CAF, coronary artery fistula; LA, left atrium; LCX, LEFT circumflex artery; OM, obtuse marginal branch.
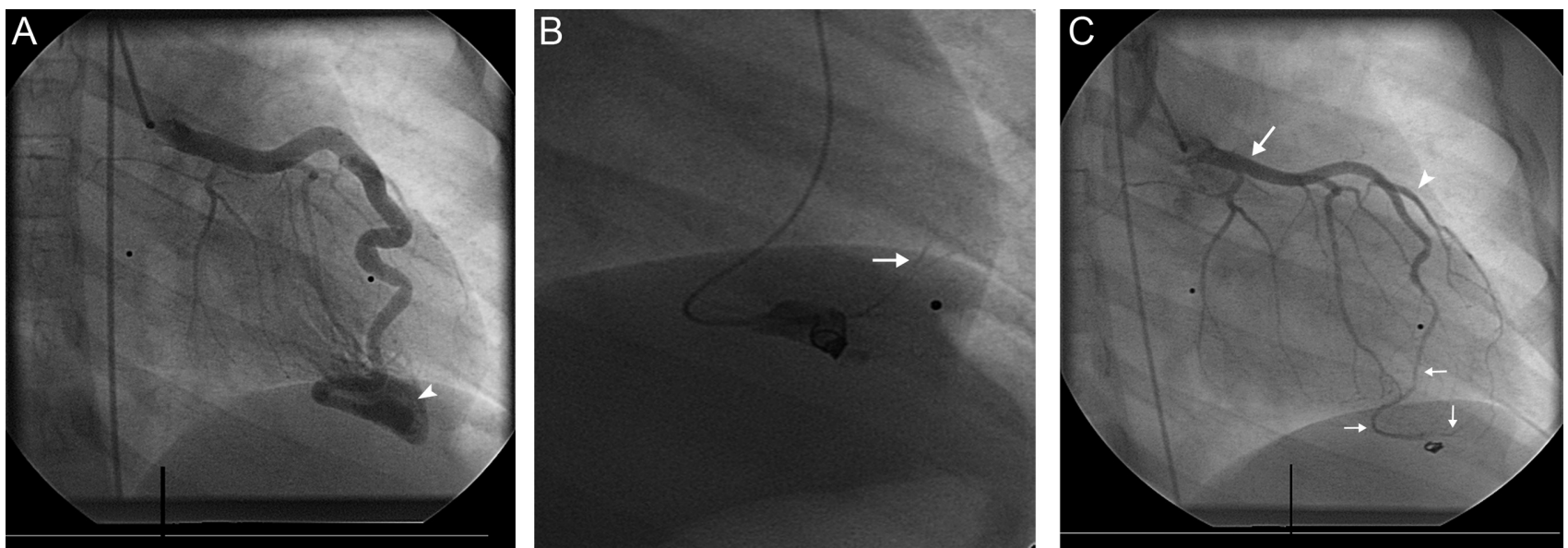

Fig. 4. Medium size distal CAF, LAD to LV. A: Six-year-old male showing medium-sized distal CAF from LAD to LV (arrow). B: Immediate post coil angiogram shows preserved small distal coronary branches from distal LAD just proximal to the coil (arrow). C: Follow-up at 6 months shows complete closure of the fistula. The proximal conduit LAD and LCA has uniform

(Fig. 2). She remained asymptomatic on oral Coumadin for 1 year and aspirin (ASA) indefinitely. Follow-up coronary angiography at 2 months and 1 year showed reduced caliber of proximal LCX conduit coronary artery (Fig. 2) with distal LCX occlusion up to the prominent proximal run-off vessel (second $\mathrm{OM}$ branch). Interestingly, there was evidence of recanalization of the distal thrombosis with thread like vessels and revascularization of the occluded OM branch. An intra vascular ultrasound (IVUS) study showed diffuse intimal thickening of proximal conduit LCX artery decrease in size towards normal (arrow), substantial decrease in distal LAD conduit coronary artery size up to the proximal run-off vessel-diagonal branch (arrow head) with preserved blood supply to small distal coronary branches (small arrows). CAF, coronary artery fistula; LAD, left anterior descending artery; LCX, left circumflex artery; LCA, left coronary artery.

(Fig. 2). Remaining 3 patients (\#13-15) had medium size distal CAF; ages 10, 6, and 2 years with successful distal coil occlusion in all. Follow up angiograms showed; uniform decrease in size of proximal conduit coronary arteries, prominent proximal run-off coronary branches, without evidence of discrete stenosis or thrombosis in all three patients (Figs. 3-5). Interestingly, there was evidence of preserved flow to distal small coronary branches with thread-like distal conduit coronary artery (Figs. 3 and 4). Furthermore in patient \#15 during CAF closure the small postero-lateral 

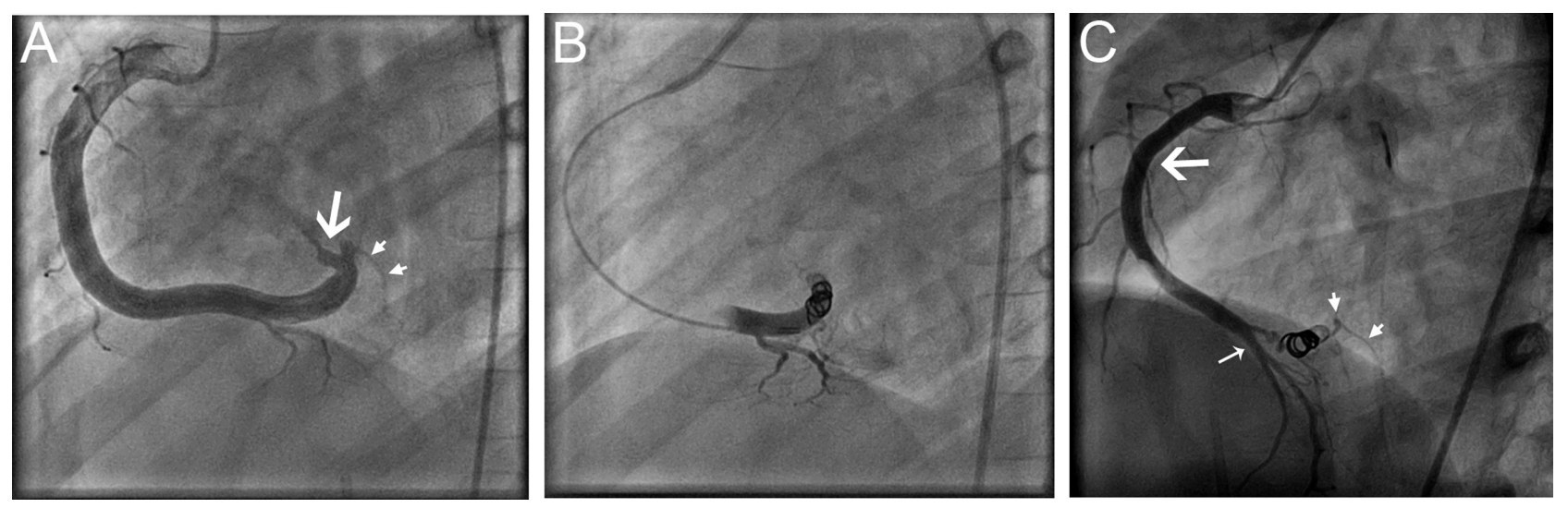

Fig. 5. Medium-size distal CAF, RCA to RV. A: A 2-year-old male with medium-sized distal fistula-RCA to RV (arrow). Adjacent to the fistula opening is a small posterior-lateral coronary branch (arrow heads). B: Post occlusion angiogram shows absence of flow to the small posterior-lateral branch distal to the coil. C: Follow-up 5 years later shows mild decrease in conduit RCA size (arrow) and adequate run-off from posterior descending artery branches just proximal to the coil (small arrow). There is revascularization (arrow heads) of the small posterior-lateral coronary branch distal to the coil. CAF, coronary artery fistula; LCA, left coronary artery; RV, right ventricle; RCA, right coronary artery.

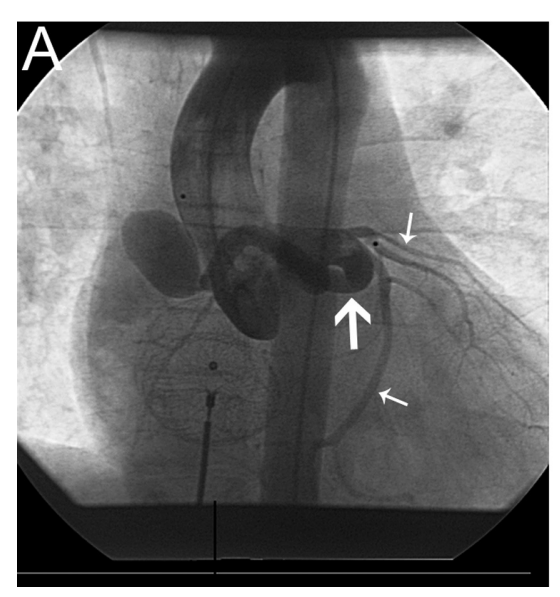

Fig. 6. Large proximal LCA to RA. A: Eleven-year-old female with large proximal CAF from LCA to the RA. There are normal LCX and LAD branches distal to the fistula (small arrows). B: Angiogram showing distal coil occlusion (arrow) of the fistula with stagnant contrast in the residual fistula stump. C: Six-year

branches were likely occluded due to inadvertent proximal placement of the coil (Fig. 5). The patient however, remained asymptomatic and follow-up angiogram showed revascularization of the occluded branches likely due to recanalization through the coil (Fig. 5).

Three of 7 patients (\#11-12) in IG had proximal CAF with successful distal TCC. Two of them were available for follow-up. A 11-year-old female (\#10) with left coronary artery (LCA) to LA large proximal fistula had distal fistula TCC. At 6-years follow-up angiogram revealed thrombosis of distal fistula segment (proximal to the coil) and interestingly there was evidence of revascularization/angiogenesis beyond the coils. The proximal fistula segment was patent follow-up angiogram shows reduced size of the proximal fistula stump with areas of discrete stenosis (small arrows), distal neovascularization-angiogenesis (arrow heads). CAF, coronary artery fistula; LCA, left coronary artery; LCX, left circumflex; LAD, left anterior descending; RA, right atrium.

partially; there was significantly decreased fistula size with evidence of diffuse intimal thickening and discrete stenosis by angiography (Fig. 6). A 3-day-old infant (\#11) had a large right coronary artery (RCA) to right ventricle (RV) proximal fistula. She presented with heart failure after birth and had SC at 5 days of age (Fig. 7). Attempted proximal ligation caused ischemic changes prompting the surgeons to ligate the CAF distally at the drainage site. Follow-up angiogram at 9 years showed occlusion of the proximal portion of the long fistula up-to the origin of RCA and no extension of thrombus to adjacent main RCA. The right aortic sinus was moderately dilated with mild aortic insufficiency and the RCA was diffusely hypoplastic (Fig. 7). 

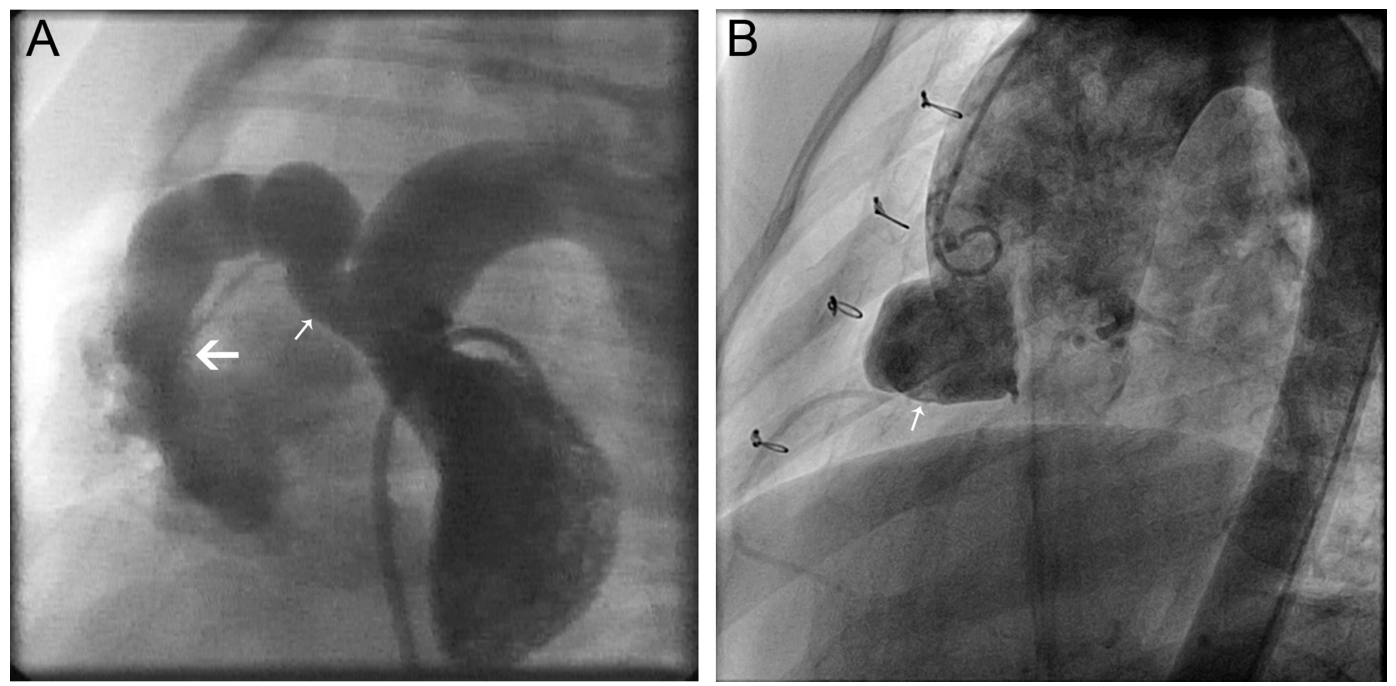

Fig. 7. Large proximal CAF from RCA to RV. A: Two-day-old infant with CHF due to large (nearly size of the aorta) proximal CAF from the RCA to RV. Large arrow indicates site of distal SC. The right coronary sinus was dilated (small arrow). B: Follow-up at 12 years of age shows thrombosis from the distal SC site up to the dilated coronary sinus (small arrow) and mild diffusely hypoplastic right coronary artery (arrow). CAF, coronary artery fistula; RCA, right coronary artery; RV, right ventricle; SC, surgical closure.
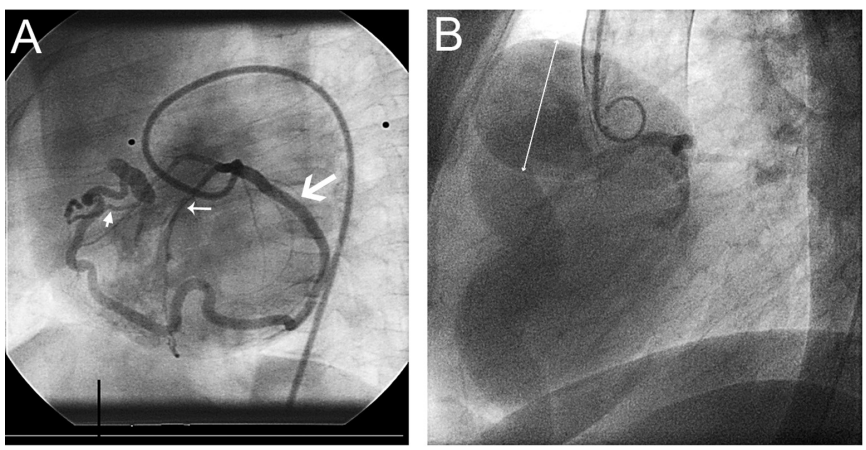

Fig. 8. Three patients with untreated large distal type CAF (nonintervention group). A: Three-month-old male with large distal CAF from LCX draining into the RV (large arrows). The proximal circumflex (conduit or feeding artery) is slightly more than twice the size of normal LAD (small arrow). Note the contribution from a branch of LAD to the fistula (arrow head). B: A large (giant) tortuous RCA to RV distal fistula in a 56-

In the NIG 6 of 9 patients were available for followup. Two with small $(\# 2,4)$ and one medium-sized proximal CAF (\#5) remained asymptomatic. Three had large distal type CAF. A 4-month-old male infant (\#7) had a large complex LCX to RV distal fistula with contributions from left anterior descending (LAD) and RCA (Fig. 8). He remained asymptomatic at 8 years of age with normal exercise stress test and was lost to follow-up. A 56-year-old female (\#8) with large (gigantic) RCA to RV distal fistula (Fig. 8) refused any form of intervention except observation on ASA. She had normal myocardial perfusion scan. She has remained
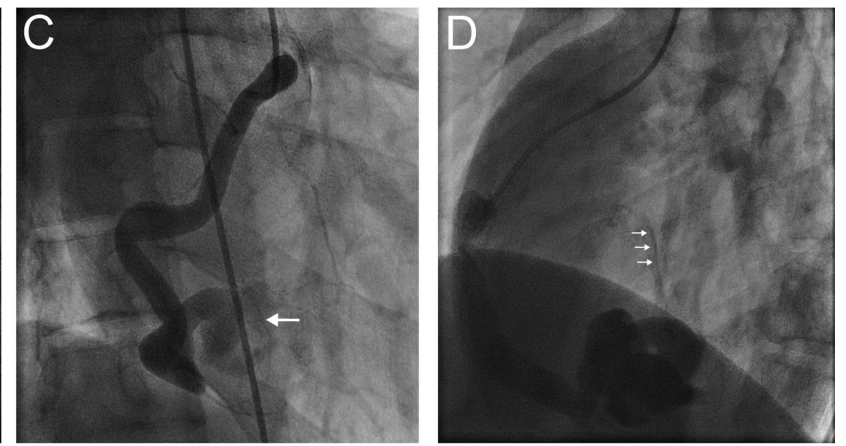

year-old female (larger than aorta at its origin-arrow). C,D: A large RCA to RV distal fistula (arrow) in a 16-year-old male. There is persistent conduit RCA dilatation at 10 years followup angiogram and evidence of tiny flow (arrows) into RV indicating spontaneous partial fistula closure. CAF, coronary artery fistula; RV, right ventricle; RCA, right coronary artery; LCX, left circumflex; LAD, left anterior descending. 


\section{DISCUSSION}

Coronary artery fistula although rare is the commonest congenital coronary anomaly with multifaceted presentation in relation to size, symptoms and age of the patient posing considerable challenge regarding the best management approach. Small CAF remain asymptomatic with potential for spontaneous closure in longterm follow-up and therefore does not require intervention [1-3]. Most medium to large CAF can be symptomatic in adults but remain asymptomatic in children $[1,4,5]$. Increased age at intervention, especially $>20$ years of age (TCC or SC) was associated with major adverse outcomes [5]. Therefore, elective closure of CAF in childhood despite absence of symptoms is advocated to prevent major complications later in life. The eventual goal of treating CAF is to provide longterm event-free survival. Conversely, several case reports and long-term studies continue to report adverse coronary events such as discrete stenosis, acute and chronic thrombosis causing myocardial infarction and perfusion defects with traditional management $[6-8,10-$ 15]. Cheung et al. to date has the most extensive followup data; 21 of 41 patients underwent coronary angiograms and all were asymptomatic on follow-up. Four had distal coronary artery complete thrombosis or were threadlike, 2 had complete thrombosis up to the proximal stump with distal collateral vessels [6]. More recently, Children's Hospital Boston published their experience with cohort of 76 patients. They identified 11 patients $(15 \%)$ to have long-term adverse coronary outcome with 9 of them having evidence of thrombosis causing MI and angina. The clinical predictors were older age at diagnosis, smoking tobacco, diabetes, systemic hypertension, and a sole angiographic feature of CAF drainage into coronary sinus [14]. These studies however failed to elucidate the risk factors for thrombosis and provided no alternative solution in managing CAF. This study with review of literature and our earlier published classification based on size and anatomy of CAF (as proximal and distal), clearly provide basis for understanding the risk factors for adverse coronary events and expose the complexity and controversies in the management of CAF.

\section{Proximal CAF}

Patients with proximal CAF are felt to be relatively at low risk for adverse events following closure from thrombosis and remodeling of the residual fistula segment as there are no normal coronary branches arising from the fistula (Fig. 1). If left untreated they have the potential to increase in size and develop symptoms from coronary steal, spontaneous thrombosis, aneurysmal dilatation leading to rupture and death [1]. They can undergo proximal or distal closure and would develop thrombosis in the residual fistula segment up to the proximal run-off major epicardial artery with out progression of thrombus to normal major coronary artery. All three patients in the present series with proximal fistula had distal closure. On follow-up data available in two patients; they had reduction is residual fistula size with discrete intimal stenosis in one (Fig. 5) and thrombosis of the residual fistula up to its origin without causing occlusion of adjacent major epicardial artery (RCA) (Fig. 6). In our review of literature only two patients with proximal CAF had coronary events (Table II) due to extension of thrombus from dilated residual fistula segment and dilated corresponding coronary sinus into the major epicardial artery $[12,15]$. We therefore recommend transcatheter approach with proximal closure of the fistula with device as suggested by our earlier report [9] or SC involving proximal ligation and surgical reduction of dilated corresponding coronary sinus when feasible to confer less risk for thrombosis extension.

\section{Distal CAF}

Patients with distal CAF (Fig. 1) can only undergo distal closure at the drainage site or just distal to the normal coronary branch. The residual conduit coronary artery especially in significantly large CAF is therefore at risk for adverse coronary events from thrombosis and unfavorable remodeling from discrete intimal stenosis [1]. In adults associated co-morbid conditions (hypertension, diabetes, hyperlipidemia, smoking, etc.) may hasten the process of procoagulation and thrombosis [14]. The dilated conduit coronary artery remodels by significant reduction in its size to conform to adjacent normal coronary branches as there is substantial reduction in flow following closure. One patient with large distal fistula (Fig. 2) and 3 other patients with medium-sized distal fistulas had moderate decrease in proximal conduit coronary artery size diffusely towards normal without discrete coronary stenosis by angiograms (Figs. 3-5). The uniform decrease in size may signify healthy remodeling mechanism in contrast to detrimental late discrete stenosis with perfusion defects as described by Hiraishi et al. in their two patients with surgical closure of large distal CAF $(0.5$ and 4.5 years of age). Discrete stenosis and perfusion defects were noted by angiography and IVUS study on follow-up evaluation [13]. The relative size of distal CAF, size of conduit coronary artery, proximity of adjacent runoff vessels, and age at intervention may determine whether reduction in conduit coronary artery size involves favorable diffuse uniform intimal thickening vs. discrete intimal stenosis associated with perfusion defects.

Thrombosis and occlusion of a normal coronary artery following fistula closure is undeniably the most 
TABLE II. Summary of Studies Reporting Adverse Coronary Events Following Closure of Large Size CAF

\begin{tabular}{|c|c|c|c|c|}
\hline $\begin{array}{l}\text { Reported studies } \\
\text { (reference) } N=25 \mathrm{pts}\end{array}$ & Age/Sex & ${ }^{\mathrm{a} C A F}$ Size, type, site, treatment & $\begin{array}{l}\text { Symptoms, F/U duration, } \\
\text { anticoagulation }\end{array}$ & $\begin{array}{c}\text { Coronary anatomical/functional } \\
\text { evaluation }\end{array}$ \\
\hline Toyoda et al. [12] & $10 / \mathrm{F}$ & Large, Proximal, RCA to RA, SC & MI, 9th day, (-) & $\begin{array}{l}\text { Thrombosis in the dilated residual } \\
\text { segment }\end{array}$ \\
\hline Mesko et al. [11] & $14 / \mathrm{F}$ & Large, Proximal, LMCA to RA, SC & MI, $10 \mathrm{yr},(-)$ & Thrombus straddling bifurcation of LAD \\
\hline Ascoop et al. [7] & $69 / \mathrm{F}$ & Large, Distal, RCA to RA, TCC & MI, few hr, (-) & $\begin{array}{l}\text { Thrombosis RCA occluding acute } \\
\text { marginal branch }\end{array}$ \\
\hline Kharouf et al. [10] & $48 / \mathrm{F}$ & Large, Distal, LCX to CS, TCC & MI, 4 days, $(-)$ & Thrombosis occluding entire LCX \\
\hline Hamada et al. [15] & 26/M & Large, Distal, LAD to RV, SC & MI, 9 mo, $(-)$ & Complete LAD occlusion \\
\hline \multirow[t]{3}{*}{ Gowda et al. [9] } & 9/M & Large, Distal, LAD to RV, SC & MI, $40 \mathrm{yr},(-)$ & Complete LAD occlusion \\
\hline & $5 / \mathrm{M}$ & Large, Distal, LCX to CS, SC & $\begin{array}{l}\text { MI, early post-operative } \\
\text { period, }(+)\end{array}$ & $\begin{array}{l}\text { Ischemic EKG changes, elevated enzymes, } \\
\text { low ventricular function-presumed } \\
\text { LCX thrombosis. }\end{array}$ \\
\hline & $56 / \mathrm{F}$ & Large, Distal, RCA to RV, SC & MI, 10 months, $(+)$ & Complete RCA thrombosis. \\
\hline \multirow[t]{2}{*}{ Hiraishi et al. [13] } & $5 / \mathrm{M}$ & Large, Distal RCA to LV, SC & Dyspnea, 5 yr, $(+)$ & $\begin{array}{l}\text { Marked reduction in size of RCA, } \\
\text { intimal thickness with discrete } \\
\text { stenosis and perfusion defects. }\end{array}$ \\
\hline & $0.5 / \mathrm{M}$ & Large, Distal RCA to RV, SC & Asymptomatic, $9 \mathrm{yr},(-)$ & $\begin{array}{l}\text { Regressed aneurysmal sites in RCA } \\
\text { with irregular vessel wall, } \\
\text { perfusion defects. }\end{array}$ \\
\hline${ }^{\mathrm{b}}$ Cheung et al. [6] & $2 \mathrm{pts}^{\mathrm{c}}$ & Presumed Distal fistula & Asymptomatic & $\begin{array}{l}\text { Both patients had entire CA thrombosis } \\
\text { up to the proximal stump with distal } \\
\text { collaterals }\end{array}$ \\
\hline${ }^{\mathrm{b}}$ Cheung et al. [6] & $4 \mathrm{pts}^{\mathrm{c}}$ & Presumed Distal fistula & Asymptomatic & 4 pts had proximal CA dilatation, distal thrombosis \\
\hline Valenete et al. [14] & $45 / \mathrm{F}$ & Large, Distal, LCX to CS, SC & $\mathrm{MI}, 4 \mathrm{yr},(+)$ & Extensive mural thrombus in mid LCX \\
\hline${ }^{b}$ Valenete et al. [14] & 6 pts & Size and drainage undetermined & MI in 4 pts, $(+)$ & $\begin{array}{l}\text { Documented intracoronary thrombus in } \\
2 \text { pts with angina }\end{array}$ \\
\hline Fahey et al. [8] & $0.1 / \mathrm{F}$ & Large, Distal, RCA to RV SC & Asymptomatic, $2 \mathrm{yr},(+)$ & $\begin{array}{l}\text { Thrombosis of entire RCA, revascularization } \\
\text { with thread like vessels. }\end{array}$ \\
\hline Current study & $15 / \mathrm{F}$ & Large, Distal LCX to RV, TCC & $\mathrm{MI},<24 \mathrm{hr},(+)$ & $\begin{array}{l}\text { Distal LCX thrombosis with occlusion of } \\
3^{\text {rd }} \text { OM branch }\end{array}$ \\
\hline
\end{tabular}

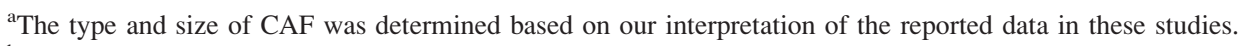

${ }^{\mathrm{b}}$ Detailed descriptions of all patients in these studies were not available for accurate interpretation.

${ }^{\mathrm{c}}$ Age undetermined.

$(-) /(+)$, Absent/Present; CAF, Coronary artery Fistula; CS, Coronary sinus; EKG, Electrocardiogram; F/U, Follow-up; LAD, Left anterior descending coronary artery; LCX, Left circumflex artery, LV, Left ventricle; LMCA, Left main coronary artery; MI, Myocardial infarction; RV, Right ventricle, RA, Right atrium; RCA, Right coronary artery; RPA, Right pulmonary artery; SC, Surgical closure; TCC, transcatheter closure.

feared and poorly understood consequence of CAF closure. The process of revascularization and remodeling following coronary occlusion can involve growth of new capillary branches from existing blood vessels, called angiogenesis and or increase in luminal diameter of an existing artery termed arteriogenesis [16]. Conceivably, these are the mechanism of revascularization observed in three patients following fistula closure (Figs. 2, 5, 6). On the basis of experience with cohort from Cleveland clinic, we were the first to report that large size, distal type CAF, and older age at closure may be at higher risk for thrombosis following CAF closure [9]. Our current study with review of literature identified 25 patients with reported coronary artery events following CAF closure. Data available in only 16 patients to evaluate size and type of CAF, clearly found 14 of these to have large size and distal CAF (Table II). We had four patients with intervention for distal CAF (all 4 were $<16$ years of age). Only one patient with large size distal CAF had an acute coronary event due to thrombosis extending into a normal coronary branch (Fig. 2). Remaining three patients with medium size distal fistula had significant uniform reduction in conduit coronary artery size without adverse coronary events (Figs. 3-5). Although patient numbers are small, these observation suggest closure of medium-sized distal CAF at a younger age may perhaps have favorable remodeling of conduit coronary artery and confer less risk for early or late thrombosis. In contrast, the decision to close a large (significantly dilated) distal CAF and timing of closure based on age continues to be an enigma. Table III summarizes coronary events based on age following closure of large distal CAF. Unambiguously, older patients with large distal CAF were at higher risk for coronary events following closure. Surprisingly younger patients with large distal CAF were also at risk for coronary events (Table III). These findings are at odds with conventional recommendation of CAF closure at less than 20 years of age [4,5] and our earlier published 
TABLE III. Summary of Studies Reporting Presence or Absence of Adverse Coronary Events Following Treatment of Large Distal Type CAF Based on Age

\begin{tabular}{|c|c|c|c|c|}
\hline \multicolumn{2}{|c|}{ Total number of patients $N=14$} & Age $<20$ years & Age $>20$ years & Total number of coronary events \\
\hline Treatment method & $\begin{array}{c}\text { IG SC or TCC } N=11 \\
\text { NIG } N=3\end{array}$ & $\begin{array}{l}N=5 \\
N=2\end{array}$ & $\begin{array}{l}N=6 \\
N=1\end{array}$ & $\begin{array}{l}\text { All } 11 \text { patients had coronary events } \\
\text { No coronary events at last follow-up }\end{array}$ \\
\hline
\end{tabular}

IG, intervention group; NIG, nonintervention group; SC, surgical closure; TCC, transcatheter closure.

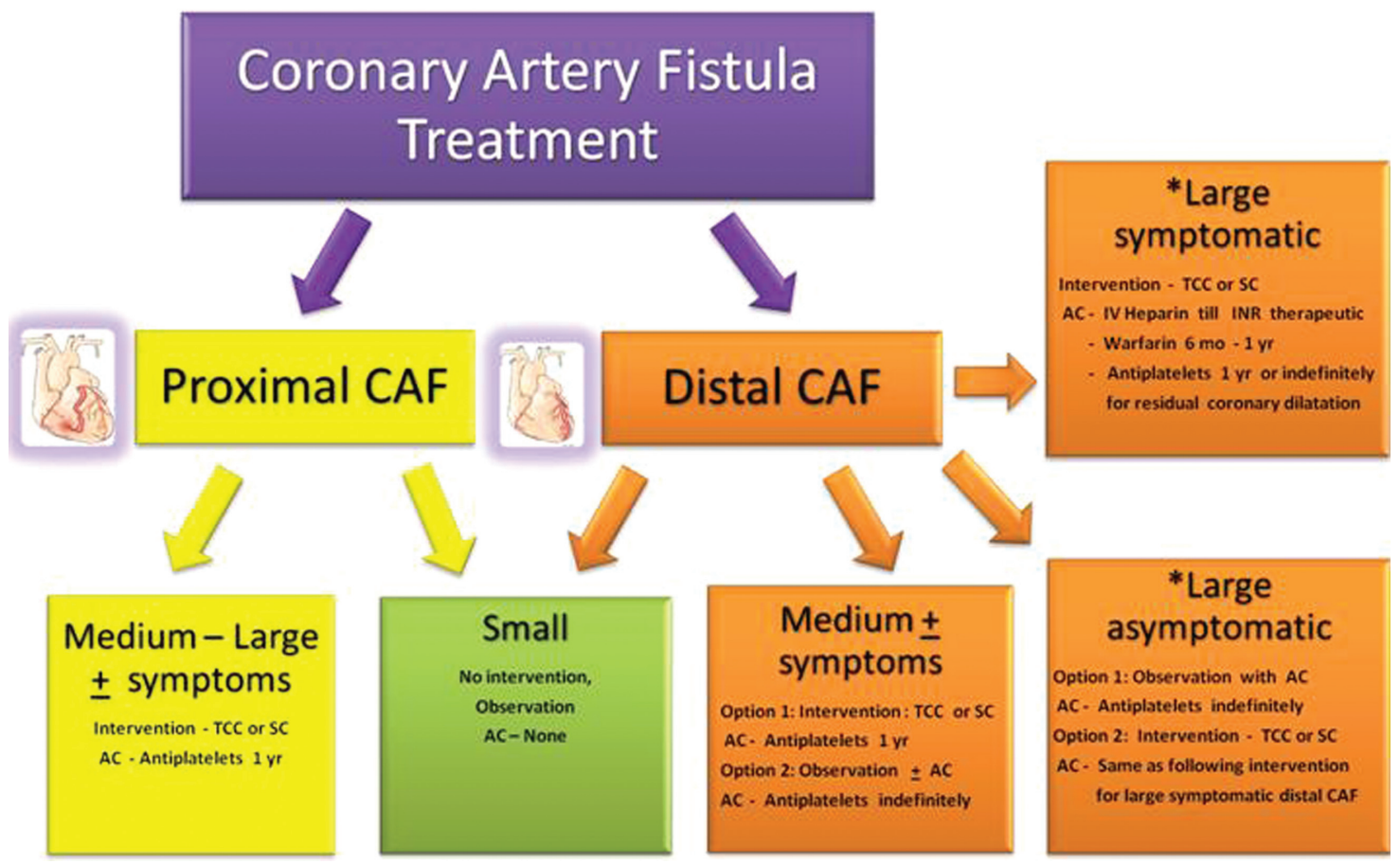

Fig. 9. CAF treatment overview with anticoagulation.

AC: Anticoagulation; IV: Intravenous; Mo: Months; SC: Surgical closure, TCC: Transcatheter closure; Yr: Year; \pm : Presence or Absence

*High risk for coronary events following closure, decision to treat based on immediate and life long risk of one option over the other.

recommendation to possibly close large distal CAF at a younger age [9]. Perhaps closure of large distal fistula at young age may not be as "protective" as hoped, but closure may still be indicated if it is felt there is a real risk for acute or late problems such as coronary steal, heart failure, rupture, endocarditis, etc. The additional confounding feature is that the natural history of untreated large distal CAF patients is not well understood. Incidentally, in this study three of our patients with large distal CAF without intervention (NIG) aged $0.3,16$, and 56 years (Fig. 8) have been asymptomatic without adverse coronary event on last follow-up at ages of 8,20 , and 62 years, respectively. Thus, nonintervention with anticoagulation may be a definite option for asymptomatic significantly large distal CAF irrespective of age.

The limitations of our study are small sample size and incomplete coronary anatomical, functional studies, and anticoagulation data on follow-up. The rarity of this disorder and treatment in specialized centers contribute to small sample size and inadequate follow-up. The definition of coronary fistula size is arbitrary. Absence of normal coronary size angiographic data in children and small patient number with CAF is the major limitation to accurately assess and classify $\mathrm{CAF}$ based on size. This review specifically highlights large size and distal CAF to be at risk for coronary events post closure irrespective of the age of the patient and thus our modified approach to management of CAF is summarized in Fig. 9. We continue to recommend medical observation and no intervention for small size, proximal and distal CAF. All proximal CAF that are moderate or large in size with or without symptoms probably should be closed, ideally as close to the origin of the fistula from the coronary tree as possible. The decision whether to intervene in patients with 
distal CAF that are medium to large remain controversial. Intervention at a younger age for medium-sized distal CAF may be recommended due to favorable remodeling mechanism. However, for large size distal CAF with significantly dilated conduit coronary arteries (irrespective of age) there appears to be a high risk for adverse coronary events following intervention. Thus nonintervention with assiduous follow-up and long-term anticoagulation may be a rational alternative. The decision to treat may best be made on the assessment of immediate and lifelong risk of one option over the other. Future studies involving multiple centers specifically assessing these risk factors for an optimal treatment approach is warranted. Until then, "To close or not to close a large distal CAF" will remain a conundrum.

\section{CONCLUSIONS}

Post CAF treatment sequelae include thrombosis and $\mathrm{MI}$, remodeling with revascularization and decrease in conduit coronary artery size towards normal. The large size, distal type of CAF may be at highest risk for coronary events post closure. The optimal treatment approach to various morphologies of CAF at various ages remains to be determined.

\section{ACKNOWLEDGEMENTS}

We would like to thank Paul Webster and Elizabeth Goodman for their tireless effort in obtaining pervious angiograms for this manuscript. We also thank the entire cath lab staff for their support and technical assistance during these procedures.

\section{REFERENCES}

1. Latson LA. Coronary artery fistulas: How to manage them. Catheter Cardiovasc Interv 2007;70:110-116.

2. Schleich JM, Rey C, Gewillig M, Bozio A. Spontaneous closure of congenital coronary artery fistulas. Heart 2001;85:E6.
3. Sherwood MC, Rockenmacher S, Colan SD, Geva T. Prognostic significance of clinically silent coronary artery fistulas. Am J Cardiol 1999;83:407-411.

4. Armsby LR, Keane JF, Sherwood MC, Forbess JM, Perry SB, Lock JE. Management of coronary artery fistulae. Patient selection and results of transcatheter closure. J Am Coll Cardiol 2002;39:1026-1032.

5. Liberthson RR, Sagar K, Berkoben JP, Weintraub RM, Levine FH. Congenital coronary arteriovenous fistula. Report of 13 patients, review of the literature and delineation of management. Circulation 1979;59:849-854.

6. Cheung DL, Au WK, Cheung HH, Chiu CS, Lee WT. Coronary artery fistulas: long-term results of surgical correction. Ann Thorac Surg 2001;71:190-195.

7. Ascoop AK, Budts W. Percutaneous closure of a congenital coronary artery fistula complicated by an acute myocardial infarction. Acta Cardiol 2004;59:67-69.

8. Fahey JT, Asnes J. Coronary recanalization due to presumed thrombosis following surgical ligation of a large right coronary artery to right ventricle fistula. Congenit Heart Dis 2008;3:295298.

9. Gowda ST, Latson LA, Kutty S, Prieto LR. Intermediate to long-term outcome following congenital coronary artery fistulae closure with focus on thrombus formation. Am $\mathrm{J}$ Cardiol 2011;107:302-308.

10. Kharouf R, Cao QL, Hijazi ZM. Transcatheter closure of coronary artery fistula complicated by myocardial infarction. J Invasive Cardiol 2007;19:E146-E149.

11. Mesko ZG, Damus PS. Myocardial infarction in a 14-year-old girl, ten years after surgical correction of congenital coronary artery fistula. Pediatr Cardiol 1998;19:366-368.

12. Toyoda Y, Yamaguchi M, Ohsima Y, et al. [A surgical case of right coronary artery-right atrial fistula complicated with postoperative myocardial infarction]. Kyobu Geka 1999;52:326331.

13. Hiraishi S, Misawa H, Horiguchi Y, et al. Effect of suture closure of coronary artery fistula on aneurysmal coronary artery and myocardial ischemia. Am J Cardiol 1998;81:12631267.

14. Valente AM, Lock JE, Gauvreau K, et al. Predictors of longterm adverse outcomes in patients with congenital coronary artery fistulae. Circ Cardiovasc Interv 2010;3:134-139.

15. Hamada M, Kubo H, Matsuoka H, Kokubu T, Oosuga Y, Joh T. Myocardial infarction complicating surgical repair of left coronary-right ventricular fistula in an adult. Am J Cardiol 1986;57:372-374.

16. Semenza GL. Vasculogenesis, angiogenesis, and arteriogenesis: mechanisms of blood vessel formation and remodeling. J Cell Biochem 2007;102:840-847. 